\title{
The role of the Coulomb interaction in the formation of superconducting and pseudogap states in cuprate metal-oxides
}

\author{
E.A. Pashitskii and V.I. Pentegov \\ Institute of Physics of the National Academy of Scienses of Ukraine, 46 Nauki Ave., Kiev, 03028, Ukraine \\ E-mail: pashitsk@iop.kiev.ua
}

Received September 1, 2005

\begin{abstract}
It is shown that the key role in the mechanism of high- $T_{c}$ superconductivity in the layered cuprate metal-oxides with anisotropic quasi-two-dimensional electronic spectrum and $d$-wave symmetry of the superconducting order parameter is played by the retarded screened Coulomb interaction and many-body correlations. We argue that the pseudogap observed in these materials is the anisotropic dielectric gap, which appears due to the electron-hole pairing on the flat portions of the Fermi surface in the vicinity of the extended saddle points in the quasiparticle spectrum. This gap coexists with the superconducting gap and is partially suppressed by scattering of current carriers on the charged point defects. The suppression of dielectric gap is analogous to the suppression of superconducting gap by magnetic impurities in «gapless» superconductors. The complete destruction of the pseudogap by charged impurities is assumed to increase $T_{C}$ significantly.
\end{abstract}

PACS: 74.72.-h

Keywords: high- $T_{c}$ superconductivity, many-body correlations, preudogap.

\section{Introduction}

The retardation effects stemming from the exchange of collective charge density excitations with quasiacoustic dispersion (acoustic plasmons) should exert a strong influence on the screened Coulomb interaction between quasiparticles in layered cuprate metal-oxides. These excitations appear both due to the quasi-two-dimensional nature of the band spectrum and as a result of the strong anisotropy of the Fermi velocity in the plane of cuprate layers. The latter is the consequence of the extended saddle points in the quasiparticle spectrum, which lead to the appearance of the square-root Van Hove singularity in the density of states in the vicinity of the Fermi level [1-4].

By virtue of the Kramers - Kronig relation for the inverse dielectric function $\varepsilon_{e}^{-1}(\mathbf{q}, \omega)$, the matrix element of the retarded screened Coulomb interaction may be represented as

$$
\tilde{V}_{c}(\mathbf{q}, \omega)=\frac{V_{c}(\mathbf{q})}{\varepsilon_{e}(\mathbf{q}, \omega)}=V_{c}(\mathbf{q})\left\{1+\int_{-\infty}^{\infty} \frac{d \omega^{\prime} S_{\mathrm{pl}}\left(\mathbf{q}, \omega^{\prime}\right)}{\omega-\omega^{\prime}+i 0}\right\},
$$

where $V_{c}$ is the bare Coulomb interaction and $S_{\mathrm{pl}}$ is the spectral function of the charge density fluctuations (plasmons). As we have shown in $[5,6]$, the low-angle inelastic scattering of current carriers on the long-wave acoustic plasmons leads to the suppression of static Coulomb repulsion $\tilde{V}_{c}(\mathbf{q}, 0)$ at small momentum transfer $\mathbf{q}$ and, eventually, to the appearance of effective interelectron attraction in the $d$-wave Cooper channel. The many-body Coulomb correlations (local field effects), taken into account through introduction of the Coulomb three-point vertex, enhance this attraction and provide for high values of the critical temperature $T_{C}$ of superconducting transition, even in the absence of any other interactions in the system.

\section{Formation of the anisotropic superconducting state in cuprate metal-oxides}

We have incorporated the retardation effects into self-consistent numerical calculations of the electronic polarizability, screened Coulomb interaction, normal and anomalous self-energies. In these calculations we 
have used theoretical spectrum of the antibonding band of Y123, proposed in [7], while the normal Coulomb vertex $\Gamma_{c}$ was taken in the approximation suggested by Nambu [8] for charged Fermi liquids:

$$
\begin{gathered}
\Gamma_{c}\left(\mathbf{k}_{\|, \omega_{n}} ; \mathbf{q}_{\|}, v_{m}\right)= \\
=\frac{1}{2}\left[Z_{c}\left(\mathbf{k}_{\|}+\mathbf{q}_{\|}, \omega_{n}+v_{m}\right)+Z_{c}\left(\mathbf{k}_{\|}, \omega_{n}\right)\right],
\end{gathered}
$$

where

$$
Z_{c}\left(\mathbf{k}_{\|, \omega_{n}}\right)=1-\frac{\operatorname{Im} \Sigma_{c}\left(\mathbf{k}_{\|}, \omega_{n}\right)}{\omega_{n}},
$$

$\Sigma_{c}$ is the part of electronic self-energy produced by the Coulomb interaction, $\mathbf{k}_{\|}$and $\mathbf{q}_{\|}$are the longitudinal quasimomenta (in the plane of layers), while $\omega_{n}$ and $v_{m}$ are, respectively, the fermionic and bosonic Matsubara frequencies at finite temperatures.

To describe superconducting state one needs to calculate the anomalous self-energy, which contains both normal $\Gamma^{N}$ and anomalous $\Gamma^{A}$ vertices. As it was shown in [9] for the electron-phonon interaction, in the vicinity of the critical temperature $T_{c}$ the anomalous self-energy, linearized with respect to the order parameter, may by represented with a good accuracy by the diagram

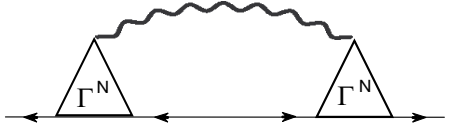

This expression, though, does not include diagrams with intersecting interaction lines, such as

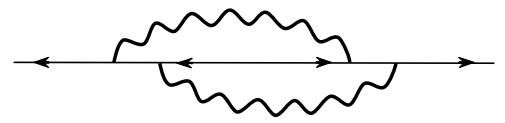

which in the case of the Coulomb interaction are not small. We took these diagrams into account introducing an additional term into the expression for the anomalous self-energy. This term represents the sum of the series of ladder diagrams, such as

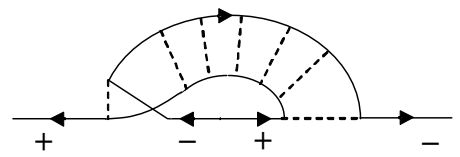

where the exact retarded interaction is approximately replaced by the screened Coulomb interaction, averaged over momenta and energy.

Accordingly, the system of equations for superconducting order parameter may by written as

$$
\Phi\left(\mathbf{k}_{\|}, \omega_{n}\right)=-\frac{T}{N^{2}} \sum_{\mathbf{k}^{\prime}, \omega_{m}}\left[K_{1}\left(\mathbf{k}_{\|}, \omega_{n} ; \mathbf{k}^{\prime} \|, \omega_{m}\right)+K_{2}\left(\mathbf{k}_{\|}, \omega_{n} ; \mathbf{k}_{\|}^{\prime}, \omega_{m}\right)\right] \Phi\left(\mathbf{k}_{\|}^{\prime}, \omega_{m}\right)
$$

where

$$
\begin{aligned}
& K_{1}\left(\mathbf{k}_{\|, \omega_{n}} ; \mathbf{k}_{\|}^{\prime}, \omega_{m}\right)=\left\langle\tilde{V}_{c}\left(\mathbf{k}-\mathbf{k}^{\prime}, \omega_{n}-\omega_{m}\right)\right\rangle_{q_{z}}\left|\Gamma\left(\mathbf{k}_{\|, \omega_{n}} ; \mathbf{k}_{\|}^{\prime}, \omega_{m}\right) G\left(\mathbf{k}_{\|}^{\prime}, \omega_{m}\right)\right|^{2}, \\
& K_{2}\left(\mathbf{k}_{\|}, \omega_{n} ; \mathbf{k}_{\|}^{\prime}, \omega_{m}\right)=V_{H}\left(\mathbf{k}_{\|}-\mathbf{k}_{\|}^{\prime}, \omega_{n}-\omega_{m}\right)\left|G\left(\mathbf{k}_{\|}^{\prime}, \omega_{m}\right)\right|^{2},
\end{aligned}
$$

and interaction $V_{H}$ is defined as

$$
V_{H}=\frac{U^{2} \Pi^{(0)}}{1-U \Pi^{(0)}} ; U=\left\langle\tilde{V}_{c}(\mathbf{q}, \omega)\right\rangle_{\mathbf{q}, \omega} ;
$$

here the angular brackets $\langle\ldots\rangle_{q_{z}}$ and $\langle\ldots\rangle_{\mathbf{q}, \omega}$ designate, respectively, the averages over the momentum $q_{z}$ along the $c$ axis, perpendicular to the plane of layers $a b$, and over the transferred momentum $\mathbf{q}$ and frequency $\omega$. The polarization operator $\Pi^{(0)}$ is calculated with exact Green functions, but with vertex $\Gamma$ taken equal to unity.

The concentration dependencies of the largest eigenvalue $\Lambda$ of the kernel of Eq. (7), obtained numerically for the $d_{x^{2}-y^{2}}$-wave symmetry of the order parameter at various temperatures, are shown in Fig. 1,a. The critical temperature of superconducting transition, which corresponds to $\Lambda=1$, is shown in Fig. $1, b$ in function of the doped holes' concentration per unit cell $x$. The value $x=x_{0}$ corresponds to the coincidence of the Fermi level with the extended saddle points.

As we can see, the effective attraction in the $d$-wave channel, originating in the exchange of charge density excitations and enhanced by many-body Coulomb correlations, produces sufficiently high critical temperatures at optimal doping level and provides a qualitatively correct description of the experimentally observed concentration dependence of $T_{c}$.

Notice the abrupt drop in $T_{c}$ when the Fermi level approaches the saddle point. This behavior, which was predicted before in [10], is the consequence of strong coupling effects in conjunction with the square-root 

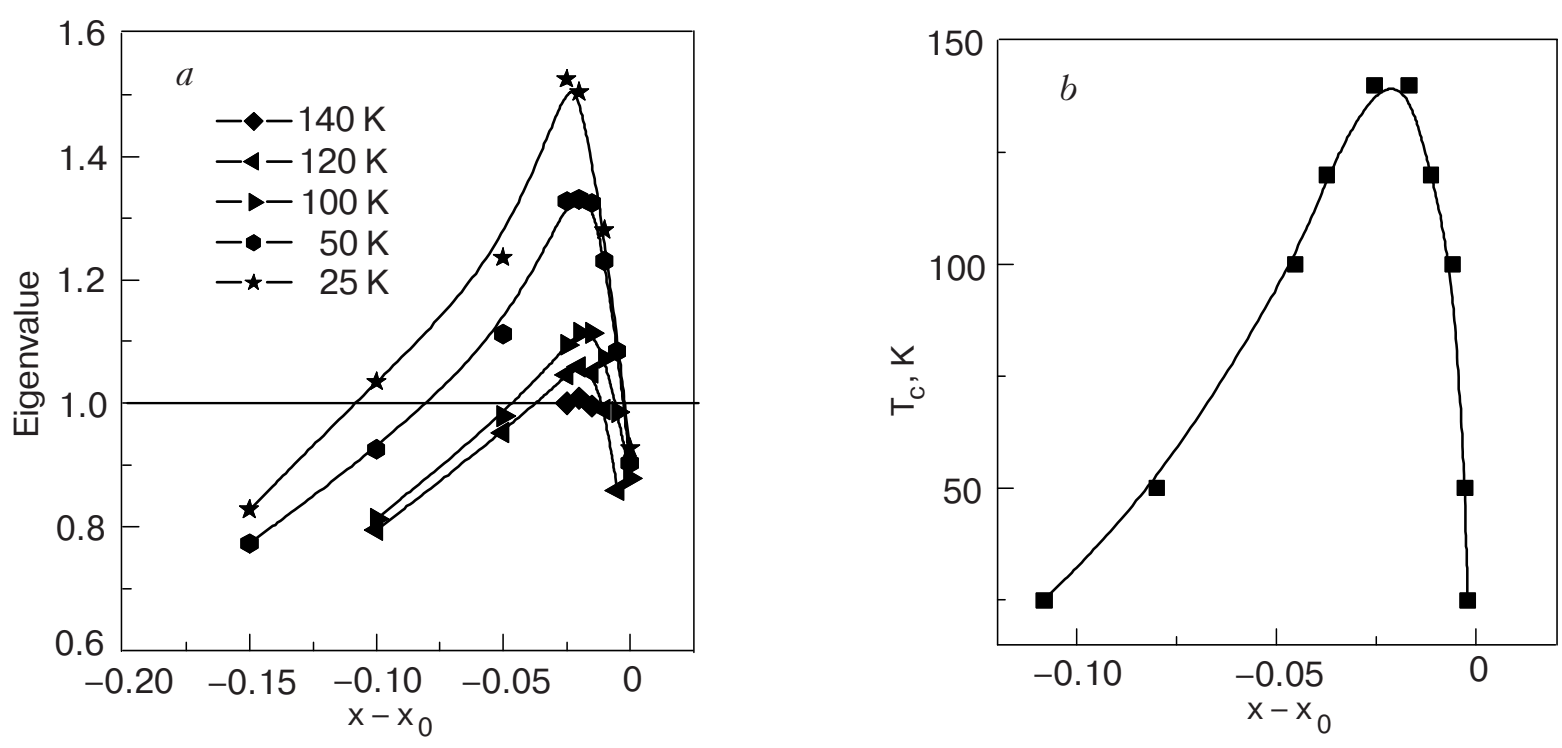

Fig. 1. The largest eigenvalue of the kernel of Eq. (7) for various temperatures ( $a$ ) and the critical temperature of superconducting transition $(b)$ in the $d_{x^{2}-y^{2}}$-wave channel in function of the concentration $x$ of the doped holes. The value $x_{0}$ corresponds to the Fermi level crossing of the saddle point.

singularity in the density of states, possessed by the band spectrum with extended saddle points.

Indeed, the anisotropic density of states, or inverse group velocity of quasiparticles, which depends on the energy $\xi$ and azimuth angle $\theta$ on the almost circular (in the $a b$ plane) cylindrical Fermi surface, may be modeled as

$$
v(\xi, \theta)=v_{+}(\xi)+v_{-}(\xi) \cos 4 \theta,
$$

where

$$
v_{ \pm}(\xi)=\frac{1}{2}\left[v_{1}\left(\mu_{0}\right) \operatorname{Re} \sqrt{\frac{\mu_{0}}{\mu+\xi}} \pm v_{2}\right],
$$

$\mu$ is the Fermi level distance from the extended saddle point and $\mu_{0}$ is the energy scale for the existence of the square-root singularity in the density of states.

Utilizing this simple approximation and taking into account the renormalization due to the strong coupling effects, we may write an approximate equation for the critical temperature $T_{c 0}$ of the transition into superconducting state with c $d_{x^{2}-y^{2}}$-wave symmetry of the gap $\Delta(\theta)=\Delta_{d} \cos 2 \theta$ :

$$
\begin{gathered}
1=\frac{W_{1}}{16(1+\lambda(\mu))}\left\{3 v_{1} \int_{-\mu}^{\Omega} \frac{d \xi}{\xi} \sqrt{\frac{\mu_{0}}{\mu+\xi}} \text { th } \frac{\xi}{2 T_{c 0}}+\right. \\
\left.+v_{2} \int_{-\Omega}^{\Omega} \frac{d \xi}{\xi} \operatorname{th} \frac{\xi}{2 T_{c 0}}\right\} .
\end{gathered}
$$

Here $\lambda(\mu) \equiv \lambda_{1} \sqrt{\mu_{0} / \mu}+\lambda_{2}$ is dimensionless renormalization constant, dependent on the isotropic part of the retarded interaction, $W_{1}$ is the matrix element of interaction in the $d_{x^{2}-y^{2}}$-channel, and $\Omega$ is characteristic energy of this interaction. When $\mu$ approaches zero with increased carrier concentration the square-root singularities in (13) are cancelled out, and the critical temperature is defined by the expression [10]

$$
T_{c 0} \approx \mu\left(\frac{3 W v_{1}}{\lambda_{1}}\right)^{2} \text { for } \mu \rightarrow 0 .
$$

The $T_{c 0}$ dependence on $\mu$, derived from (13), is shown in Fig. 2, $a$ (curve 1-1').

\section{Nesting and «excitonic» pairing in the presence of charged impurities}

For quasiparticles on the nearest flat portions of the Fermi surface, stretched along the «banks» of the extended saddle, the energy satisfy the nesting condition $\varepsilon(\mathbf{k})=-\varepsilon\left(\mathbf{k}+\mathbf{q}_{0}\right)$, where the nesting vector $q_{0}$ is small $\left(q_{0}<\pi / a\right)$ and depends on the doping level of the crystal. The Coulomb repulsion between electrons with transferred momentum $q_{0}$, described by the matrix element $W_{0}$, corresponds then to the effective attraction between an electron and a hole. As the result, the anisotropic dielectric gap may appear on these flat portions of the Fermi surface due to the instability of the ground state with respect to the coherent electron-hole (excitonic) pairing, i.e., to the phase transition into the state of excitonic dielectric [11]. The equation for this «excitonic» gap may be written, in the absence of a superconducting gap, as: 

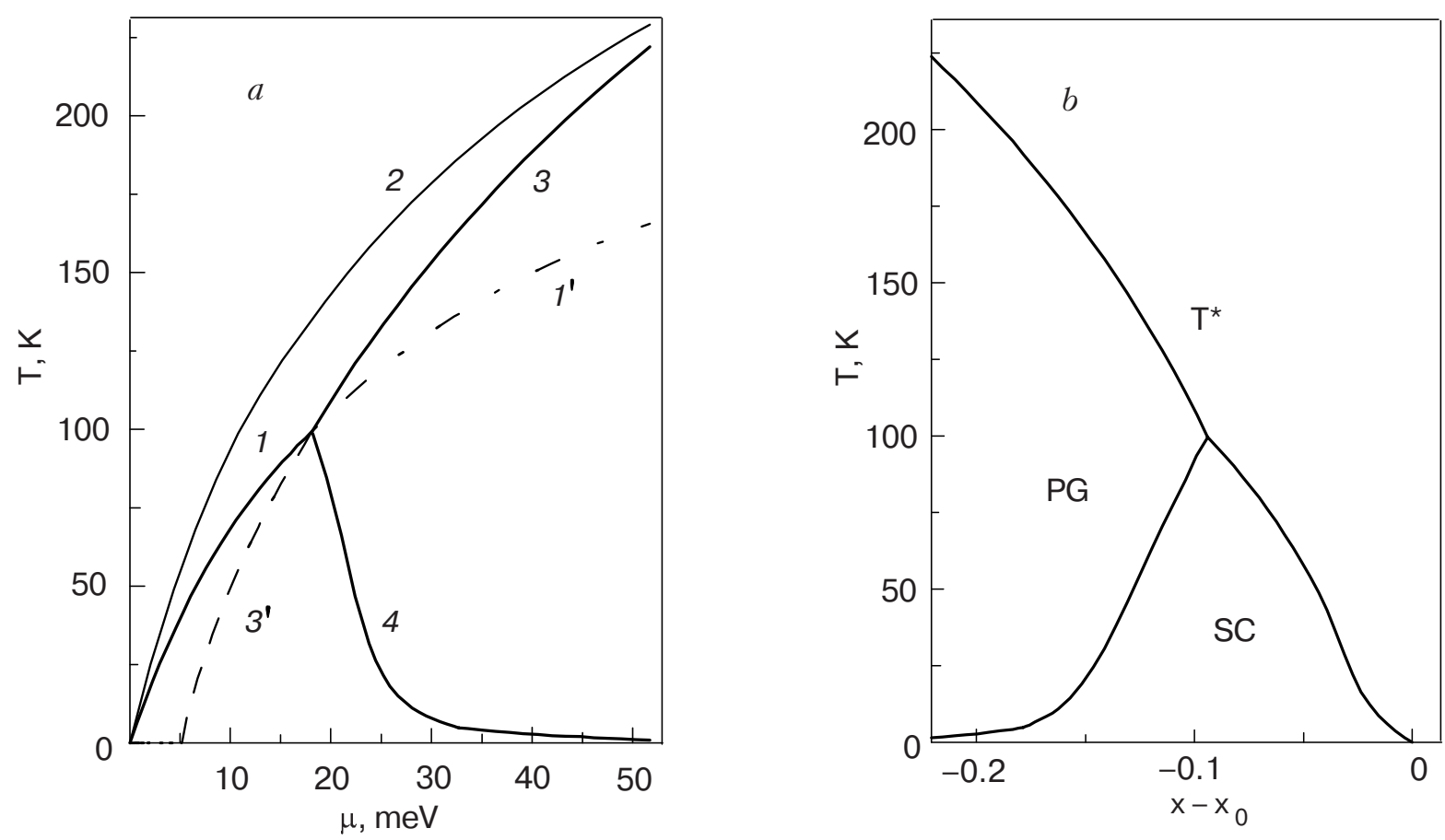

Fig. 2. (a) The dependencies of $T_{c}$ and $T_{d}$ on $\mu$; the numbers near the curves are explained in text. (b) The phase diagram of the superconducting and pseudogap states.

$$
1=\frac{W_{0} v_{1}\left(\mu_{0}\right)}{2[1+\lambda(\mu)]} \int_{-\mu}^{\Omega} d \xi \frac{\sqrt{\mu_{0} /(\mu+\xi)}}{\sqrt{\xi^{2}+\Sigma_{0}^{2}(T)}} \text { th } \frac{\sqrt{\xi^{2}+\Sigma_{0}^{2}(T)}}{2 T} \text {. }
$$

It should be noted that the dielectric gap $\Sigma_{0}$ has a purely electronic nature and does not imply a Pierls instability of the crystal lattice because the nesting vector $q_{0}$, in general, is not commensurate with the reciprocal lattice period $2 \pi / a$.

The critical temperature of the transition into the excitonic dielectric state $T_{d 0}(\mu)$, given the condition $W_{0}>W_{1} / 2$, is shown in Fig. 2, $a$ with curve 2 .

Nevertheless, the Eq. (15) does not account for the influence of charged impurities, the doped oxygen ions in particular, on the «excitonic» gap $\Sigma$ and critical temperature $T_{d}$ through the pair-breaking process. This effect is due to the opposite signs of the amplitudes of scattering of the electrons and holes on the charged impurities and is similar to the influence of the magnetic impurities on superconducting gap and $T_{c}$ in the «gapless» superconductors with singlet Cooper pairing [12]. The lowering of $T_{d}$ in comparison with $T_{d 0}$ in cuprates due to such pair braking in the process of the elastic scattering on the oxygen ions may be accounted for using the equation, obtained in [12]:

$$
\ln \left(T_{d 0} / T_{d}\right)=\psi(1 / 2+\rho / 2)-\psi(1 / 2),
$$

where $\psi$ is the digamma function. The dimensionless parameter $\rho=\hbar /\left(\pi T_{d} \tau_{c}\right)$ in our case is proportional to the inverse time $\tau_{c}^{-1}=N \sigma_{c^{v}}{ }_{F}$ of the Coulomb scattering of quasiparticles with Fermi velocity $v_{F}$ in the 2D cuprate layers on charged impurities with concentration $N$ (per unit area of the layer). The cross section of the 2D Coulomb scattering on a screened impurity in the Born approximation equals

$$
\sigma_{c}=\frac{4 \pi^{2} e^{4} m^{2} r_{0}^{2}}{\varepsilon_{0} \hbar^{4} k_{F}} \frac{1}{\sqrt{1+4 r_{0}^{2} k_{F}^{2}}},
$$

where $\varepsilon_{0} \approx 100-200$ is the dielectric function of the lattice, $\hbar k_{F}$ is the Fermi momentum, and $r_{0}$ is the screening length.

The curve $3-3^{\prime}$ in Fig. 2, $a$ shows the dependence of $T_{d}$ in function of $\mu$, calculated with the use of (16). The position of the Fermi level $\mu$ with respect to the saddle point depends on the concentration of the doped holes, which is equal to the concentration of the dopant oxygen atoms $n$.

The matrix elements $W_{0}$ and $W_{1}$ are calculated through the Fourier expansion of the static screened Coulomb matrix element on the Fermi surface, and with account of the Coulomb vertices $\Gamma$ (see [13]).

\section{The phase diagram}

The dielectric and superconducting gaps exist on virtually the same portions of the Fermi surface in the vicinity of saddle points thus strongly influencing each other. In particular, when $T_{c 0}<T_{d}$, the critical temperature of superconducting transition $T_{c}$ for $\Sigma \neq 0$ is given by the equation (cf. Eq. (13)) 


$$
\begin{gathered}
1=\frac{W_{1}}{16[1+\lambda(\mu)]}\left\{3 v_{1} \int_{-\mu}^{\Omega} d \xi \frac{\sqrt{\mu_{0} /(\mu+\xi)}}{\sqrt{\xi^{2}+\Sigma^{2}\left(T_{c}\right)}} \text { th } \frac{\sqrt{\xi^{2}+\Sigma^{2}\left(T_{c}\right)}}{2 T_{c}}+\right. \\
\left.+v_{2} \int_{-\Omega}^{\Omega} \frac{d \xi}{\xi} \operatorname{th} \frac{\xi}{2 T_{c}}\right\} .
\end{gathered}
$$

In Fig. 2, $a$ the curve 4 shows the dependence of $T_{c}$ on $\mu$ in the region, where $T_{c 0}<T_{d}$. In the same time, in the region, where $T_{c 0}>T_{d}$, the Eq. (18) leads to the former result for $T_{c}$, given by (13), i.e., $T_{c}=T_{c 0}$ (curve 1).

It should be noted that far from the critical concentration of charged impurities given by the expression $\tau_{c 0}=2 \gamma \hbar /\left(\pi T_{d 0}\right)$, the temperature dependence of $\Sigma(T)$ is described by the usual BCS equation, while in the vicinity of the critical concentration one should use the dependence obtained in [12]

$$
\Sigma(T)=\pi \sqrt{2\left(T_{d}^{2}-T^{2}\right)} .
$$

The critical temperatures of superconducting $\left(T_{c}\right)$ and dielectric $\left(T_{d}\right)$ transitions are shown in Fig. 2,b in function of the concentration of doped holes. The calculated phase diagram qualitatively agrees with the experimentally obtained one for the cuprate superconductors (cf. [14]), if $T_{d}$ is identified with characteristic temperature $T^{*}$ of the pseudogap state.

Finally, in Fig. 3 the calculated phase diagram is compared with the experimental concentration dependences of $T_{c}$ and $T^{*}$ (see [15]). Here the charge carrier concentration $x_{\max }$ corresponds to the maximal critical temperature $T_{c \max }$ of superconducting transition. We should emphasize again, that for the sake of simplicity we have limited the calculation to include only one coefficient of the Fourier expansion of the interaction matrix element for each of the interaction channels $\left(W_{0}\right.$ и $\left.W_{1}\right)$, and have also utilize the model expression for the angular dependence of the electronic Fermi velocity (12). Nevertheless, even this simplified model allows for sufficiently good agreement with experiment with actually only one «free» parameter the dielectric constant of the lattice $\varepsilon_{0}$.

\section{Conclusion}

In conclusion, in the framework of the model considered we assume that the pseudogap, observed in the cuprate metal-oxide, is actually a dielectric gap in quasiparticle spectrum, partially suppressed by the charged oxygen ions. It should be noted that according to our model the total suppression of the dielectric (excitonic) gap $\Sigma$ would lead to a substantial increase in the maximal $T_{c}$ (up to the maximal value of $T_{c 0}$ ).

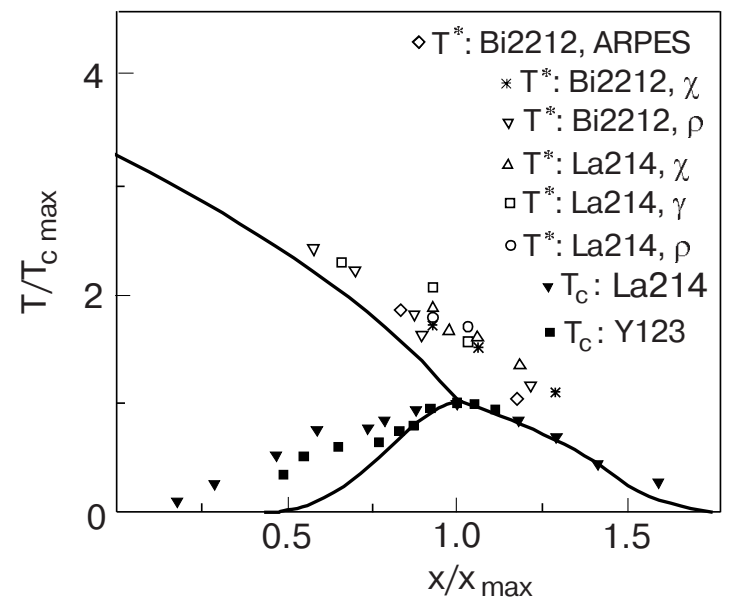

Fig. 3. Experimental concentration dependencies of $T_{C}$ and $T^{*}$ [15]. The solid line corresponds to the phase diagram, calculated for the value $\varepsilon_{0}=100$.

Thus, we may suggest a possible way of enhancing $T_{c}$ in cuprates. Suppose we managed to achieve additional doping of a high- $T_{c}$ cuprate with donors and acceptors with optimally chosen concentrations (analogously to the compensated semiconductors), which preserves the charge carriers concentration. Since the pair-breaking effect does not depend on the sign of the impurity charge, the only result of such doping would be the suppression of pseudogap, and, consequently, the enhancement of $T_{c}$. This increase in superconducting transition temperature is illustrated in Fig. 4.

Finally, note that the electron-hole pairing on the flat portions of the Fermi surface near the extended saddle points should be accompanied by the appearance of incommensurate charge-density waves in the directions of the main crystallographic axes. Due to the dielectric gap suppression by charged impurities these charge-density waves should degrade into the

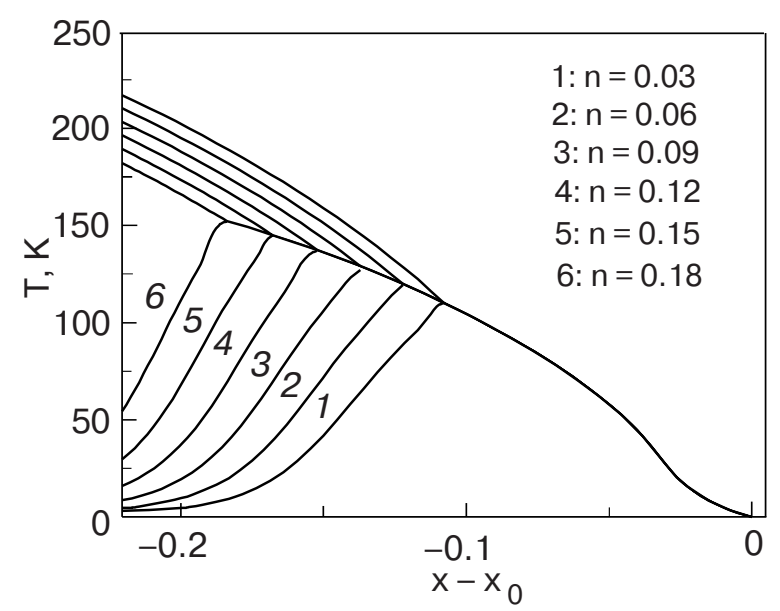

Fig. 4. The enhancement of $T_{C}$ with addition of compensated dopants. Curves $1-6$ correspond to the change in the dopant concentration $n$ from 0.03 to 0.18 ions per unit cell. 
metastable periodic stripe structure, coexisting with the superconducting state.

1. A.A. Abrikosov, J.C. Campuzano, and K. Gofron, Physica C214, 73 (1993).

2. A.A. Abrikosov, Physica C214, 107 (1993).

3. A.A. Abrikosov, Physica C222, 191 (1994).

4. A.A. Abrikosov, Physica C244, 243 (1995).

5. E.A. Pashitskii and V.I. Pentegov, JETP Lett. 72, 439 (2000)

6. E.A. Pashitskii and V.I. Pentegov, in Models and Methods of High- $T_{c}$ Superconductivity: Some Frontal Aspects, J.K. Srivastava and S.M. Rao (eds.), Nova Science Publishers, New York (2003), Vol. 1, p. 299.

7. O.K. Andersen, O. Jensen, A.I. Liechtenstein, I.I. Mazin, Phys. Rev. B49, 4145 (1994).
8. Y. Nambu, Phys. Rev. 117, 648 (1960).

9. O. Dolgov and E.G. Maksimov, Proceedings of the P.N. Lebedev Physical Institute (in Russian) 148, 3 (1982).

10. E.A. Pashitskii, V.I. Pentegov, and E. Abraham, JETP Lett. 67, 495 (1998).

11. L.V. Keldysh and Yu.V. Kopaev, Fiz. Tverd. Tela 6, 2791 (1964).

12. A.A. Abrikosov and L.P. Gor'kov, Zh. Eksp. Teor. Fiz. 39, 1781 (1960).

13. E.A. Pashitskii and V.I. Pentegov, Fiz. Nizk. Temp. 27, 140 (2001) [Low Temp. Phys. 27, 103 (2001)].

14. A. Damascelli, Z. Hussain, and Z.-X. Shen, Rev. Mod. Phys. 75, 473 (2003).

15. C.P. Moca and B. Janko, Phys. Rev. B65, 052503 (2002). 